DOI: 10.12957/demetra.2016.24936

\title{
Mudanças na ingestão de alimentos entre obesas após três meses de intervenção nutricional
}

\section{The changes in food intake between obese people after 3 months of nutritional intervention}

\author{
Ana Emilia da Silva Santos' \\ Anelisa Spinelli Peixoto ${ }^{1}$ \\ Helena Siqueira Vassimon ${ }^{2}$ \\ 1 Universidade de Franca, Curso de Nutrição. \\ Franca-SP, Brasil. \\ ${ }^{2}$ Universidade de Franca, Programa Pós- \\ graduação Stricto Sensu de Promoção da Saúde. \\ Franca-SP, Brasil. \\ Artigo elaborado a partir do trabalho de \\ conclusão de curso da aluna A.E.S. SANTOS, sem \\ apoio financeiro. \\ Correspondência / Correspondence \\ Helena Siqueira Vassimon \\ E-mail: helenavassimon@yahoo.com.br
}

\section{Resumo}

Avaliar mudanças da ingestão de alimentos entre obesas após 3 meses de intervenção nutricional em grupo. Vinte obesas foram convidadas para participar de 6 encontros quinzenais de 2 horas/cada, totalizando três meses de acompanhamento. Cada encontro foi elaborado baseado no Guia Alimentar para População Brasileira, documento proveniente da Política e ações em alimentação e nutrição do governo brasileiro. Antes e depois da intervenção foram avaliadas medidas antropométricas (peso, altura e circunferência da cintura), ingestão de alimentos pelo recordatório de $24 \mathrm{~h}$ e comparando com diretrizes do Guia Alimentar para População Brasileira. Apenas oito obesas finalizaram o estudo, e foi observada redução significativa da circunferência abdominal entre momento inicial $(126 \pm 9,1 \mathrm{~cm})$ e final $(119,7 \pm$ $8,0 \mathrm{~cm})$. Houve redução significativa de sódio $(1726,9 \pm 963,1$ para $1092,4 \pm 492,1 \mathrm{mg})$ e gordura trans $(3,3 \pm 2,07$ para $1,6 \pm 1,5 \mathrm{~g} /$ dia) e aumento na ingestão de niacina $(13,5 \pm 6,7 \mathrm{mg} /$ dia para $1,3 \pm 4,5 \mathrm{mg} / \mathrm{dia})$ a partir do método do recordatório de $24 \mathrm{~h}$. E comparando com as recomendações do Ministério da Saúde, observou-se que todas as obesas ao final do programa relataram adequação da ingestão de refrigerantes, óleo, sal, frutas, verduras, legumes, água e fracionamento da dieta. Conclui-se que houve redução da circunferência abdominal e melhora da ingestão de alimentos utilizando intervenção nutricional em grupo.

Palavras-chave: Ingestão de alimentos. Obesidade. Educação alimentar e nutricional. 


\section{Abstract}

This study aims to evaluate the changes in food intake between obese people after 3 months of nutritional intervention. Twenty obese were invited to participate of 6 biweekly meetings of 2 hours / each, totaling three months of follow-up. Each meeting was created using Brazilian Food intake Guideline, document from actions and politics in food and nutrition of the Brazilian government. Before and after intervention, the anthropometric measures were evaluated (weight, height and waist circumference), food intake by $24 \mathrm{~h}$ recall and comparing with the Brazilian Food intake Guideline. Only eight obese patients completed the study and a significant reduction of abdominal circumference was observed between the initial $(126 \pm 9,1 \mathrm{~cm})$ and the final $(119,7 \pm$ $8,0 \mathrm{~cm})$ moments. There was a significant reduction of sodium $(1726,9 \pm 963,1$ for $1092,4 \pm 492,1 \mathrm{mg})$ and trans fat $(3,3 \pm$ $2,07$ for $1, \pm 1,5 \mathrm{~g} /$ day $)$ and the increase of niacin intake $(13,5 \pm$ $6,7 \mathrm{mg} /$ day for $1,3 \pm 4,5 \mathrm{mg} /$ day) from the $24 \mathrm{~h}$ recall method. Comparing this with the recommendations of the Brazilian Food intake Guideline, it was observed, at the end of study, that all obese patients had an appropriate consumption of soft drink, oil, salt, fruits, greens, vegetables, water and diet fractionation. It was concluded that there were an abdominal circumference reduction and an improvement in food intake, using nutritional intervention in a group.

Key words: Eating Obesity. Food and Nutrition Education.

\section{Introdução}

$\mathrm{Na}$ atualidade, a obesidade se coloca de maneira prioritária para intervenção, em nível individual e na comunidade, como um problema de nutrição em saúde pública.'A obesidade pode ser conceituada de maneira simplificada, como uma condição de acúmulo anormal ou excessivo de gordura no organismo, levando a um comprometimento da saúde. ${ }^{1} \mathrm{O}$ grau de excesso de gordura e sua distribuição estão associados com prejuízo considerável para saúde, sendo fundamental que existam ações para prevenção, mas também o tratamento da obesidade, pois os portadores dessa condição apresentam riscos aumentados de morbidade e mortalidade. ${ }^{1}$

A obesidade surgiu como uma epidemia em países desenvolvidos e tem sido considerado importante problema de saúde pública em todos os níveis socioeconômicos e está aumentando 
nos países em desenvolvimento., ${ }^{2,3}$ Em 2008, o sobrepeso e obesidade no Brasil atingiram cerca de metade dos homens e das mulheres, excedendo em 28 vezes a frequência do déficit de peso no caso masculino e em 13 vezes no feminino. ${ }^{4}$

Segundo Consenso Brasileiro para Tratamento da Síndrome Metabólica, a adoção de uma dieta balanceada deve considerar características socioeconômico-culturais, e a redução de $5 \%$ a $10 \%$ do peso já está associada à melhoria dos níveis da pressão arterial, controle metabólico e até mesmo da mortalidade relacionada ao diabetes mellitus. ${ }^{5}$

A intervenção nutricional em grupo tem sido utilizada para melhorar hábitos alimentares de diversos grupos populacionais sem a necessidade de trabalhar com cardápios individualizados. A intervenção em grupo tem por objetivo buscar uma construção coletiva do conhecimento, valorizando a participação de todos e não só passando conteúdo, mas preocupando-se com que cada um tenha sua visão crítica para suas escolhas alimentares, assim empoderando-os. ${ }^{6}$ A literatura traz vários resultados de estudos com intervenção nutricional em grupo. Estudo realizado em ambulatório com objetivo de avaliar a estratégia de intervenção nutricional em grupo de 40 pessoas obesas concluiu que houve melhora com redução em torno de $5 \%$ do peso e circunferência abdominal e ingestão calórica após reuniões quinzenais por 6 meses abordando temas de emagrecimento e hábitos de vida saudáveis. ${ }^{7}$ Outro estudo com mesmo objetivo e usando metodologia participativa comparou atendimentos individuais (total de 3 consultas) com atendimentos em grupo (total de 6 encontros). Foram 33 participantes e observaram que as principais dificuldades para perda de peso relatadas foram quanto a eventos sociais e comer fora de casa. O estudo não obteve resultados significativos de melhora da ingestão de alimentos. ${ }^{8}$

O presente estudo teve por objetivo avaliar a eficácia da intervenção nutricional em grupo por três meses para mudança de ingestão de alimentos em mulheres obesas.

\section{Métodos}

Trata-se de estudo longitudinal aprovado pelo Comitê de Ética em Pesquisa sob número 21847513.3.0000.5495. Todas as participantes assinaram o Termo de Consentimento Livre e Esclarecido (TCLE) antes do início da pesquisa.

A amostra foi intencional, sendo as participantes convocadas por intermédio da Clinica de Nutrição de Universidade localizada na cidade de Franca, interior do Estado de São Paulo (Brasil), que atende gratuitamente a população. A pesquisadora utilizou-se da lista de espera para atendimento na clínica para selecionar participantes. Seguindo a lista, a pesquisadora ligava e explicava sobre a pesquisa, e após concordância, era agendado dia e horário para primeiro encontro para assinatura do TCLE, esclarecimentos da pesquisa e aplicação de protocolo inicial. 
Os critérios de inclusão foram: mulheres, com idade entre 18 e 60 anos, portadoras de obesidade grau I, II ou III. Os critérios de exclusão foram: mulheres obesas que participavam de outro programa de educação nutricional ou realizaram cirurgia de obesidade ou faziam uso contínuo de medicamento para emagrecimento.

Foram convidadas para participar 25 obesas, entretanto, apenas 20 iniciaram a intervenção nutricional. O motivo da recusa por parte de cinco convidadas foi indisponibilidade de horário. Das 20, apenas 8 completaram 100\% de presença nos 6 encontros enquanto 12 desistiram entre o 2o e o $3^{\underline{o}}$ encontros, relatando indisponibilidade de horário e dificuldades para locomoção. Em dezembro. todas as 8 obesas retornaram para nova avaliação

A intervenção nutricional foi iniciada com grupo de 20 mulheres obesas entre maio até julho de 2013. Os encontros tinham frequência quinzenal durante três meses, totalizando seis reuniões quinzenais com duas horas de duração cada uma, carga horária de 12 horas. Todo o conteúdo abordado tinha como base diretrizes das políticas de alimentação e nutrição brasileiras publicadas pelo Ministério da Saúde, principalmente o documento Guia Alimentar para População Brasileira, ${ }^{9}$ rotulagem nutricional, composição de um prato saudável, dietas da moda e apresentação de documentário sobre obesidade (Muito além do peso). ${ }^{10}$ Era realizado sempre uma roda e focado na metodologia participativa, cartazes e exemplos práticos por exemplo de rótulos foram levados nas reuniões. Filme foi passado em retroprojetor para otimizar a visualização de todas participantes.

Foram coletados dados antropométricos (peso, estatura e circunferência abdominal) no primeiro dia (Momento inicial), após 3 meses de intervenção (Momento final) e após 4 meses do final da intervenção (Reavaliação). Para aferir o peso foi solicitado que o indivíduo retirasse o excesso de roupa, estivesse descalço e com os braços estendidos ao longo do corpo. Foi utilizada balança digital da marca Filizola com precisão de $100 \mathrm{~g}$ e que suporta até $200(\mathrm{~kg})$. Para aferição da estatura, foi orientado que a pessoa posicionasse os pés paralelos e as mãos ao longo do corpo, sem sapatos ou objetos na cabeça, com joelhos unidos e olhando para o horizonte, utilizando o estadiômetro vertical de parede da marca Sanny ${ }^{\circledR}$. Foi usado como método de classificação do estado nutricional o IMC segundo a OMS (1998). Por último, a circunferência abdominal (CA) foi padronizada e aferida na altura da cicatriz umbilical. A classificação da circunferência abdominal considerou risco aumentado de $88 \mathrm{~cm}$ para mulheres e $102 \mathrm{~cm}$ para homens. ${ }^{5}$

Outro dado coletado nos três momentos foi a ingestão de alimentos através do recordatório de 24h. Este método tem como objetivo quantificar todos os alimentos e bebidas ingeridas no período anterior à entrevista, que pode ser $24 \mathrm{~h}$ precedentes. Foi utilizada tabela brasileira de composição dos alimentos (TACO) e para cálculos nutricionais foi utilizado programa Diet Pro ${ }^{\circledR}$, resultando em valores de calorias, macronutrientes e micronutrientes.

Para comparar ingestão de alimentos com o recomendado pelo Guia Alimentar para População Brasileira foram utilizados os "Dez passos para alimentação saudável” (Quadro 1). Foram coletados 
dados em frequências de consumo dos itens, por exemplo: anotado a frequência do consumo de legumes, verduras e frutas por semana e depois comparado com o adequado seguindo o guia, que descreve no Passo 3 que o adequado seria 3x/dia de frutas e 3x/dia de verduras ou legumes. Os dados foram obtidos por anotações ou perguntas simples que permitiram respostas diretas sobre cada um dos itens.

Quadro 1. "Dez passos para alimentação saudável”

\begin{tabular}{|c|c|}
\hline 1 & $\begin{array}{l}\text { Faça pelo menos três refeições (café da manhã, almoço e jantar) e dois lanches } \\
\text { saudáveis por dia. Não pule refeições. }\end{array}$ \\
\hline 2 & $\begin{array}{l}\text { Inclua diariamente seis porções do grupo de cereais (arroz, milho, trigo, pães e massas), } \\
\text { tubérculos como as batatas e raízes como a mandioca/ macaxeira/ aipim nas refeições. } \\
\text { Dê preferência aos grãos integrais e aos alimentos na sua forma mais natural. }\end{array}$ \\
\hline 3 & $\begin{array}{l}\text { Coma diariamente pelo menos três porções de legumes e verduras como parte das } \\
\text { refeições e três porções ou mais de frutas nas sobremesas e lanches. }\end{array}$ \\
\hline 4 & $\begin{array}{l}\text { Coma feijão com arroz todos os dias ou, pelo menos, cinco vezes por semana. Esse } \\
\text { prato brasileiro é uma combinação completa de proteínas e bom para a saúde. }\end{array}$ \\
\hline 5 & $\begin{array}{l}\text { Consuma diariamente três porções de leite e derivados e uma porção de carnes, } \\
\text { aves, peixes ou ovos. Retirar a gordura aparente das carnes e a pele das aves antes } \\
\text { da preparação torna esses alimentos mais saudáveis. }\end{array}$ \\
\hline 6 & $\begin{array}{l}\text { Consuma, no máximo, uma porção por dia de óleos vegetais, azeite, manteiga ou } \\
\text { margarina. Fique atento aos rótulos dos alimentos e escolha aqueles com menores } \\
\text { quantidades de gorduras trans. }\end{array}$ \\
\hline 7 & $\begin{array}{l}\text { Evite refrigerantes e sucos industrializados, bolos, biscoitos. doces e recheados, } \\
\text { sobremesas, doces e outra guloseimas como regra da alimentação. }\end{array}$ \\
\hline 8 & $\begin{array}{l}\text { Diminua a quantidade de sal na comida e retire o saleiro da mesa. Evite consumir } \\
\text { alimentos industrializados com muito sal (sódio) como hambúrguer, charque, } \\
\text { salsicha, linguiça, presunto, salgadinhos, conservas de vegetais, sopas, molhos e } \\
\text { temperos prontos. }\end{array}$ \\
\hline 9 & $\begin{array}{l}\text { Beba pelo menos dois litros ( seis a oito copos) de água por dia. Dê preferência ao } \\
\text { consumo de água nos intervalos das refeições. }\end{array}$ \\
\hline 10 & $\begin{array}{l}\text { Torne sua vida mais saudável. Pratique pelo menos } 30 \text { minutos de atividade física } \\
\text { todos os dias e evite as bebidas alcoólicas e o fumo. Mantenha o peso dentro de } \\
\text { limites saudáveis. }\end{array}$ \\
\hline
\end{tabular}


A análise estatística trata-se de apresentação dos resultados de forma descritiva, sendo qualitativos quando apresentados em frequências e porcentagens, enquanto dados quantitativos em médias e desvios-padrões. E para comparação entre momento inicial e final da intervenção nutricional, foi aplicado teste de Wilcoxon para amostras pareadas por se tratar de dados não paramétricos. Foi utilizado 5\% como nível de significância para o teste estatístico.

\section{Resultados}

Em relação aos dados antropométricos foi possível observar que uma participante classificada com obesidades grau 3 passou ao final do estudo para grau 2. Todas participantes apresentaram alto risco para doenças cardiovasculares segundo CA, entretanto, ao comparar os valores absolutos (Teste de Wilcoxon para amostras pareadas) antes e depois da intervenção foi observado redução significativa houve redução (Tabela 1).

Tabela 1. Avaliação antropométrica de obesas participantes de intervenção nutricional em grupo por três meses. Franca-SP, 2013.

\begin{tabular}{|c|c|c|}
\hline Indicadores & Inicial & Final \\
\hline Peso $(\mathrm{kg})$ & $103,1 \pm 21$ & $100,9 \pm 21$ \\
\hline Estatura (m) & $1,58 \pm 0,09$ & $1,58 \pm 0,09$ \\
\hline IMC (kg/m2) & $40,41 \pm 5,27$ & $39,98 \pm 5,45$ \\
\hline Obesidade grau 1 (n) & 1 & 1 \\
\hline Obesidade grau 2 (n) & 2 & 3 \\
\hline Obesidade grau 3 (n) & 5 & 4 \\
\hline Circunferência abdominal (cm) & $126,5 \pm 9,1^{*}$ & $121,2 \pm 8,6 *$ \\
\hline Risco elevado $(>90 \mathrm{~cm})$ & $8 / 8$ & $8 / 8$ \\
\hline
\end{tabular}

$* \mathrm{p}<0,05$

A ingestão de macronutrientes e energia a partir da análise de composição de nutrientes obtidos pelo recordatório de 24 horas não foi diferente entre momentos (Tabela 2), apesar de observar certa redução numérica da quantidade de energia. 
Tabela 2. Avaliação da ingestão energética e de macronutrientes das obesas participantes de intervenção nutricional em grupo por três meses. Franca-SP, 2013.

\begin{tabular}{|c|c|c|c|}
\hline Ingestão Alimentar & Inicial & Final & $\mathrm{p}$ valor \\
\hline Energia (kcal) & $1543,2 \pm 440,2$ & $1332,4 \pm 515,4$ & 0,26 \\
\hline Carboidrato (g) & $195,9 \pm 51,8$ & $184,0 \pm 124,4$ & 0,57 \\
\hline$(\%)$ & $52,3 \pm 16,6$ & $50,8 \pm 17,3$ & 0,89 \\
\hline \multirow[t]{2}{*}{ Lipídio (g) } & $48,0 \pm 30,4$ & $29,6 \pm 16,9$ & 0,09 \\
\hline & $25,4 \pm 10,9$ & $21,1 \pm 12,04$ & 0,40 \\
\hline \multirow[t]{2}{*}{ Proteína (g) } & $79,8 \pm 43,2$ & $82,2 \pm 27,8$ & 0,89 \\
\hline & $19,8 \pm 9,06$ & $26,1 \pm 9,3$ & 0,26 \\
\hline
\end{tabular}

Na Tabela 3, os resultados referente à qualidade da gordura obtidos também pelo Recordatório de $24 \mathrm{~h}$ refletem que houve redução da ingestão de gordura trans (Tabela 3). Por último, em relação aos micronutrientes, foi possível observar na Tabela 4 que as obesas apresentaram aumento da ingestão de niacina e redução na ingestão do sódio após intervenção nutricional em grupo.

Tabela 3. Avaliação da ingestão de gordura das obesas participantes de intervenção nutricional em grupo por três meses e reavaliação após quatro meses. Franca-SP, 2013.

\begin{tabular}{|c|c|c|c|c|}
\hline Gordura & & Inicial & Final & $\mathrm{p}$ valor \\
\hline \multirow[t]{2}{*}{ Saturada (g) } & & $14,5 \pm 12,2$ & $13,1 \pm 9,02$ & 0,78 \\
\hline & $(\%)$ & $7,5 \pm 4,4$ & $9,4 \pm 7,2$ & 0,26 \\
\hline \multirow[t]{2}{*}{ Monoinsaturada(g) } & & $13,4 \pm 8,2$ & $11,8 \pm 8,7$ & 0,12 \\
\hline & $(\%)$ & $7,1 \pm 2,8$ & $8,3 \pm 5,7$ & 0,67 \\
\hline \multirow[t]{2}{*}{ Poliinsaturada(g) } & & $8,2 \pm 5,3$ & $4,1 \pm 3,1$ & 0,07 \\
\hline & $(\%)$ & $4,5 \pm 2,5$ & $2,6 \pm 1,4$ & 0,57 \\
\hline Trans (g) & & $3,3 \pm 2,07$ & $1,1 \pm 1,2$ & 0,03 \\
\hline Relação W6/W3 (g) & & $13,4 \pm 3,4$ & $17,4 \pm 8,04$ & 0,48 \\
\hline W3(g) & & $6,6 \pm 4,6$ & $7,2 \pm 5,2$ & 0,12 \\
\hline W6(g) & & $7,6 \pm 4,9$ & $3,8 \pm 2,8$ & 0,40 \\
\hline
\end{tabular}


Tabela 4. Avaliação de micronutrientes das obesas participantes de intervenção nutricional em grupo por 3 meses. Franca-SP, 2013.

\begin{tabular}{lccc}
\hline & Inicial & Final & p valor \\
\hline Cálcio $(\mathrm{mg})$ & $230,9 \pm 153,8$ & $364,9 \pm 282,6$ & 0,40 \\
Magnésio $(\mathrm{mg})$ & $154,7 \pm 47,5$ & $170,4 \pm 60,2$ & 0,48 \\
Manganês $(\mathrm{mg})$ & $1,6 \pm 7,4$ & $1,3 \pm 8,1$ & 0,26 \\
Fósforo $(\mathrm{mg})$ & $897,3 \pm 396,7$ & $947,5 \pm 375,05$ & 0,78 \\
Ferro $(\mathrm{mg})$ & $7,8 \pm 3,9$ & $6,9 \pm 3,2$ & 0,67 \\
Sódio $(\mathrm{mg})$ & $1726,9 \pm 963,1$ & $816,7 \pm 555,9$ & 0,01 \\
Potássio $(\mathrm{mg})$ & $1518,9 \pm 588,1$ & $2046,6 \pm 655,6$ & 0,12 \\
Cobre $(\mathrm{mg})$ & $5,7 \pm 7,2$ & $6,8 \pm 8,3$ & 0,78 \\
Zinco $(\mathrm{mg})$ & $10,6 \pm 9,4$ & $8,8 \pm 4,02$ & 1,00 \\
Retinol $(\mathrm{mg})$ & $140,7 \pm 187,08$ & $61,8 \pm 86,5$ & 0,07 \\
Tiamina $(\mathrm{mg})$ & $1,1 \pm 1,6$ & $1,7 \pm 2,6$ & 0,78 \\
Riboflavina $(\mathrm{mg})$ & $1,1 \pm 1,6$ & $1,1 \pm 2,7$ & 0,67 \\
Piridoxina $(\mathrm{mg})$ & $1,5 \pm 2,7$ & $2,1 \pm 3,9$ & 0,20 \\
Niacina $(\mathrm{mg})$ & $13,5 \pm 6,7$ & $30,2 \pm 21,8$ & 0,05 \\
Vitamina C (mg) & $31,3 \pm 23,4$ & $97,2 \pm 91,3$ & 0,07 \\
Fibras $(\mathrm{mg})$ & $11,06 \pm 2,8$ & $16,2 \pm 11,7$ & 0,16 \\
\hline
\end{tabular}

Ao comparar dados relatados de frequências de consumo e recomendações do Guia Alimentar para População Brasileira foi possível observar maiores modificações da ingestão de alimentos do que o Recordatório de $24 \mathrm{~h}$. Destaca-se relato de adequação de quase todas obesas quanto ao fracionamento da dieta e consumo de óleo, refrigerantes, sal, água e frutas, verduras e legumes após a intervenção. A pesquisadora voltou na casa dessas obesas após 4 meses que havia finalizado a intervenção e observou que houve melhora ainda mais, em que todas as obesas estavam com ingestão adequadas desses itens avaliados (Tabela 5). 
Tabela 5. Comparação da ingestão de alimentos segundo algumas orientações do Guia Alimentar para População Brasileira. Franca-SP, 2013.

\begin{tabular}{lcc}
\hline \multicolumn{1}{c}{ Adequação } & inicial & final \\
\hline Fracionamento das refeições & $6 / 8(75 \%)$ & $6 / 8(75 \%)$ \\
Consumo verduras e frutas & $6 / 8(75 \%)$ & $7 / 8(87,5 \%)$ \\
Consumo de óleo mensal & $5 / 8(62,5 \%)$ & $7 / 8(87,5 \%)$ \\
Consumo de refrigerante & $5 / 8(62,5 \%)$ & $6 / 8(75 \%)$ \\
Consumo de sal mensal & $5 / 8(62,5 \%)$ & $8 / 8(100 \%)$ \\
\hline
\end{tabular}

\section{Discussão}

O estudo destaca o menor consumo de gordura trans após intervenção. Corrêa (2009) ${ }^{11}$ teve como objetivo verificar o consumo de ácidos graxos trans em alguns países e seus efeitos na alimentação avaliaram vários produtos alimentícios e observaram que alimentos industrializados são os que mais detêm elevados teores de gorduras trans. O mesmo estudo comparou consumo entre países, e os resultados mostraram estimativas de consumo individual diário de 7g nos EUA assim como na Argentina e de $5 \mathrm{~g}$ no Brasil. ${ }^{11}$ Os valores brasileiros são pouco maiores dos que foram encontrados no presente estudo. Importante salientar que após a intervenção em grupo os valores reduziram para $1 \mathrm{~g} /$ dia, reforçando a importância da educação nutricional para evitar o consumo destes alimentos que têm sido vastamente incluído no mercado.

Foi observado que a maior parte das obesas realizavam poucas refeições ao dia e após intervenção todas passaram a realizar adequadamente as cinco refeições. Blundell et al. ${ }^{12}$ evidenciaram que o fracionamento das refeições ao longo do dia ajuda a diminuir a fome e evitar uma supercompensação nas próximas refeições. Farshchi et al. ${ }^{13}$ mostraram que grupo de mulheres magras que mantiveram um período de alimentação irregular, apresentaram maior incidência de insensibilidade à insulina pós-prandial e maiores níveis de colesterol total e LDL, do que o grupo de mulheres que mantiveram alimentação regular.

Em relação à ingestão de frutas, verduras e legumes, é interessante salientar que mesmo sem observar melhora significativa em termos de fibras, pelo relato das obesas todas afirmaram estar com ingestão em frequência proposta pelo Ministério da Saúde. Outro estudo ${ }^{14}$ obteve resultados semelhantes após intervenção nutricional em 80 famílias residentes em dois bairros do distrito do Grajaú, Município de São Paulo, em 2004. O grupo intervenção compareceu em três encontros realizados na própria comunidade em semanas sucessivas, cada um com aproximadamente duas horas de duração, enquanto que o grupo controle não participou desses encontros, os quais 
foram convidados para preencher nos meses anterior e posterior a intervenção questionários de aquisição de alimentos. ${ }^{14}$ No momento inicial e final, a participação de frutas e hortaliças na alimentação das famílias foi caracterizada a partir do percentual de calorias provenientes desses alimentos no total de calorias adquiridas para consumo da família ao longo de um mês e no final da intervenção houve aumento do consumo de frutas e hortaliças em média, respectivamente, de $+1,63 \%$ e $+0,41 \%$ no grupo intervenção, diferente do controle que teve redução de - 0,01\%. ${ }^{14}$

A melhora da ingestão de nutrientes após intervenção nutricional em grupo também tem sido descrita na literatura atual. Vargas et al. ${ }^{15}$ avaliaram os efeitos de um programa de prevenção à obesidade sobre práticas alimentares de adolescentes de escolas públicas. A intervenção foi com 331 estudantes de 11 a 17 anos de $5^{\text {o }}$ e $6^{\text {o }}$ anos de duas escolas públicas estaduais de Niterói, RJ, em 2005. As escolas foram classificadas em escola de intervenção e escola de controle para comparação. Práticas alimentares foram abordadas em questionários autorrespondidos antes e após o período de intervenção: consumo de fast food, consumo de refrigerantes, substituição de refeições por lanches, consumo de frutas, verduras e legumes e tipo de alimentação consumida nos intervalos das aulas. ${ }^{15}$ $\mathrm{Na}$ linha de base participaram 185 estudantes da escola intervenção (82,2\% dos elegíveis) e 146 estudantes da escola controle (70,5\% dos elegíveis). Na fase pós-intervenção houve perda de 10,3\% dos adolescentes da escola intervenção e 27,4\% da escola controle. Não se observaram mudanças significativas nas práticas alimentares na escola controle, enquanto na escola intervenção aumentou a proporção de estudantes que relataram não consumir lanches vendidos por ambulantes (de 36,7\% para 50,6\%; $\mathrm{p}=0,02$ ) e dos jovens que relataram não substituir por lanche o almoço (de 44,5\% para 65,2\%; $\mathrm{p}<0,01$ ) ou jantar (de 38,4\% para 54,3\%; $\mathrm{p}<0,01$ ). A principal mudança favorável foi a redução na freqüência de consumo de lanches fast food na escola intervenção comparada à escola controle $(72,7 \%$ vs $54,4 \% ; \mathrm{p}=0,001)$. Mudanças favoráveis nas práticas alimentares dos adolescentes foram encontradas e estimulam a implantação de programas dessa natureza; contudo, intervenções de maior duração precisam ser implementadas e avaliadas quanto a sua efetividade. ${ }^{15}$

O estudo traz resultados positivos de redução de circunferência abdominal, pela avaliação do recordatório de 24 h houve redução significativa do consumo de sódio e gordura trans e aumento do consumo de niacina e comparando com recomendações do Guia Alimentar para População Brasileira, houve adequação da ingestão de refrigerantes, óleo, sal, frutas, verduras, legumes, água e fracionamento da dieta.

\section{Referências}

1. Organização Mundial de Saúde. Obesidade, prevenção e administração da epidemia global. Relatório de uma consulta sobre obesidade. Genève: OMS; 1998.

2. Bernardi F, Cichelero C, Vitolo MR. Comportamento de restrição alimentar e obesidade. Rev Nutr. 2005; 18(1):86-88. 
3. Popkin BM, Doak C. A epidemia da obesidade é um fenômeno no mundo todo. Rev Nutr. 1998; 56:106-114.

4. Instituto Brasileiro de Geografia e Estatística. Pesquisa de Orçamentos Familiares 2008-2009 Antropometria e estado nutricional de crianças, adolescentes e adultos no Brasil. Rio de Janeiro: IBGE; 2010. Disponível em: http://www.abeso.org.br/uploads/downloads/70/553a23f27da68.pdf

5. Brandão AP, Brandão AA, Nogueira AR, Suplicy H, Guimarães JI, Oliveira JEP. I Diretriz brasileira de diagnostico e tratamento da síndrome metabólica. Arq Bras. 2005; 84(1):22-24.

6. Brasil. Ministério da Saúde. Obesidade. Brasília: Ministério da Saúde; 2006. (Cadernos de Atenção Básica, no 12). [acesso em: 25 jul. 2016]. Disponível em: http://189.28.128.100/dab/docs/publicacoes/ cadernos_ab/abcad12.pdf

7. Moreira P, Romualdo MCS, Amparo FC, Paiva C, Alves R, Magnoni D, Kovacs C. A educação nutricional em grupo e sua efetividade no tratamento de pacientes obesos. Rev Bras Obesidade, Nutrição e Emagrecimento 2012; 6(35):216-224.

8. Guimarães NG, Dutra ES, Eliane S, Ito MK, Carvalho KMB. Adesão a um programa de aconselhamento nutricional para adultos com excesso de peso e comorbidades. Rev Nutr. 2010; 23(3):323-24.

9. Brasil. Ministério da Saúde. 10 passos para a alimentação saudável. Brasília: Ministério da Saúde; 2010. 19 p.

10. Muito além do peso. Direção: Estela Renner. São Paulo: Maria Farinha Filmes; 2012. (103 m). [acesso em: 25 jul. 2016]. Disponível em https://www.youtube.com/watch?v=8UGe5GiHCT4

11. Corrêa BMJ. Ácidos graxos trans e a alimentação moderna [trabalho de conclusão de curso]. Brasília: Universidade de Brasília; 2008.

12. Blundell JE, Burley VJ, Cotton JR, Lawton CL. A gordura dietética e do controle da ingestão de energia: Avaliar os efeitos da gordura no tamanho da refeição na saciedade pós refeição. Am J Clin Nutr. 1993; 57:773-76.

13. Farshchi H, Taylor M, Macdonald I. Frequência de refeição regular cria perfis de sensibilidade à insulina e perfil lipídico mais apropriadas em comparação com frequência da refeição irregular em mulheres saudáveis. Eur J Clin Nutr. 2004; 58:1071-74.

14. Jaime PC, Machado FMS, Westphal MF, Monteiro CA. Educação nutricional e consumo de frutas e hortaliças: ensaio comunitário controlado. Rev Saúde Publica 2007; 41(1):2-4.

15. Vargas ICS, Sichieri R, Pereira GS, Veiga G. Avaliação de programa de prevenção de obesidade em adolescentes de escolas públicas. Rev Saúde Pública 2011; 45(1):59-64

Recebido: 02/8/2016

Aceito: 18/9/2016 
